\title{
Stabilization of solitons in coupled nonlinear pendulums with simultaneous external and parametric excitations
}

\author{
A. Jallouli, N. Kacem*, N. Bouhaddi \\ FEMTO-ST Institute, UMR 6174, Applied Mechanics Department, \\ University of Franche-Comté, UBFC, 24 Chemin de l'Épitaphe, \\ F 25000, Besançon, France
}

\begin{abstract}
The influence of an external harmonic excitation on the intrinsic localized modes of a chain of nonlinear pendulums is numerically investigated. We show, in particular, how the existence and stability domains of solitons are modified when the coupled pendulums are simultaneously subjected to external and parametric excitations. This stabilization mechanism opens the way towards the control of the energy localization phenomena in damped nonlinear periodic lattices for efficient energy transport applications.
\end{abstract}

Keywords: Soliton, Coupled Pendulums, Nonlinear Localization, Simultaneous Excitations.

\section{Introduction}

In the past few years, there were several studies focused on the dynamic behavior of coupled nonlinear oscillators and the most used model is the array of coupled pendulums. This model and its properties are used to investigate many physical phenomena like the propagation of solitons on fluid surfaces [1, 2, 3], parametric generation of spin waves in ferro- and antiferromagnets [4, 5], solitons in Josephson junctions [6] and microelectromechanical and intrinsic localized modes in nanoelectromechanical systems (MEMS and NEMS) [7].

The array of coupled pendulums has been studied for more than thirty years and from different points of view. Ikeda et al. [8] investigated the intrinsic localized modes for a small array of

*. Corresponding author. Tel. : +33 381666702 ; fax : +33381666700.

Email address: najib.kacem@femto-st.fr (N. Kacem) 
oscillators (two and three pendulums) subjected to horizontal sinusoidal excitation. The frequency response was obtained by solving the derived equation of motion using Van Der Pols method. The authors showed the effect of the linear coupling spring an added imperfection on the soliton and they proved these results on an array of Duffing oscillators [9] subject to an external excitation. For a large number of oscillators, Ivancevic et al. [10] transformed the equations of motion of an array of coupled oscillators to one Sine-Gordon equation and reviewed the essential dynamics of a nonlinear excitation in living cellular structures. Khomeriki et al. [11] studied the tristability of a chain of pendulums driven periodically in one end and free at the other end.

There are two types of excitation : an external excitation $[12,13]$ by applying torques to the pendulums and a parametric excitation $[14,15,16,17]$ by moving periodically the support of the pendulums. The first type of excitation was simulated by Braiman et al. [12] on a chain of coupled damped pendulums with a free end boundary condition. The authors showed that when the chain is homogeneous (all pendulums have the same length), the oscillations become chaotic. However, their motion becomes ordered when some impurity is added. The notion of impurity was also tested numerically [13] on an array of 128 pendulums. The parametric excitation was studied numerically by Alexeeva et al. [14] and experimentally by Chen et al. [15]. The authors showed that "long" impurities can extend the region of stability of the system and short impurities are responsible of oscillatory instabilities.

In term of nonlinear energy localization, the equations of motion of the system have been transformed into a nonlinear Schrodinger equation (NLS) using the multiple scale method in order to analyze numerically $[16,17]$ and experimentally $[18]$ the interactions between impurities and solitons in parametrically driven coupled pendulums and the impurity proved their ability to stabilize a chaotic oscillator. Nevertheless, to our knowledge, these phenomena have not been investigated when the pendulums are subjected to simultaneous excitations, even though beneficial effects have been demonstrated in term of collective dynamics for coupled nonlinear oscillators under simultaneous external and parametric excitations [19] and in terms of performances for micro and nano-sensors under simultaneous primary and superharmonic resonances [20, 21].

In this paper, we derived the equations of motion describing the nonlinear dynamics of an array of coupled pendulums under simultaneous external and parametric excitations. In order to 
investigate the intrinsic localized modes, the system of nonlinear equations is transformed into a Schrodinger equation which has been numerically solved using the Runge-Kutta method. Numerical simulations are performed on a typical design of 301 coupled pendulums proving that the region of existence and stability of solitons can be significantly increased by means of simultaneous parametric and external excitations.

\section{Design and model}

The considered system, depicted in Figure 1, is composed of an horizontal axle A. Along this axle, at equally spaced intervals, there are $N_{\text {pen }}$ equal pendulums. Each pendulum consists of a rigid rod, attached perpendicularly to the axle, with a mass $m$ at the end. At rest, all the pendulums point down the vertical. $a$ is the distance between two pendulums, $g$ is the gravity acceleration, $\theta_{n}$ is the angle between the $n^{\text {th }}$ pendulum and the downward vertical, $k_{L}$ is the linear torque constant. The kinetic and potential energy of the system can be written as :

$$
\begin{gathered}
V=\sum_{n} \frac{1}{2} k_{L}\left(\theta_{n}-\theta_{n+1}\right)^{2}+\frac{1}{2} k_{L}\left(\theta_{n}-\theta_{n-1}\right)^{2}-m g l\left(\sin \left(\theta_{n}\right)+A_{e} \cos (2 \omega t)\right) \\
T=\sum_{n} \frac{1}{2} m v_{n}^{2}
\end{gathered}
$$

The potential energy $V$ includes two parts : the strain energy due to the elongation of the spring and the gravitational potential energy; $T$ is the kinetic energy due to the velocity $v_{n}$ of the moving mass :

$$
\overrightarrow{v_{n}}=\overrightarrow{\dot{r}_{O A}}+\vec{\omega} \times \overrightarrow{r_{A P}}
$$

where

$$
\begin{array}{ll}
\overrightarrow{\dot{r}_{O A}}=A_{e} \omega_{e} \sin (\omega t) \vec{y} & \vec{\omega}=\dot{\theta}_{n} \vec{z} \\
\overrightarrow{r_{A P}}=l\left(\sin \left(\theta_{n}\right) \vec{x}+\cos \left(\theta_{n}\right) \vec{y}\right) &
\end{array}
$$

we applied the Lagrange formulation

$$
\frac{d}{d t}\left(\frac{\partial L}{\partial \dot{\theta}_{n}}\right)-\frac{\partial L}{\partial \theta_{n}}=Q_{n}
$$




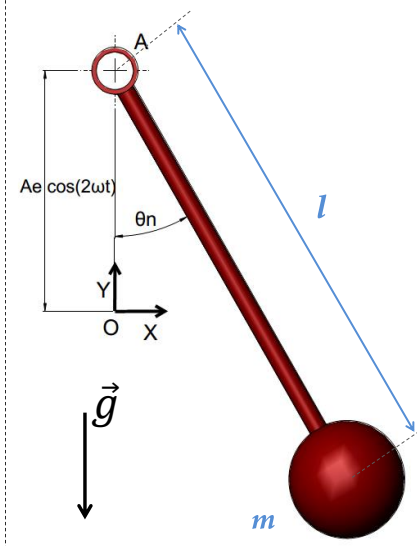

(a)

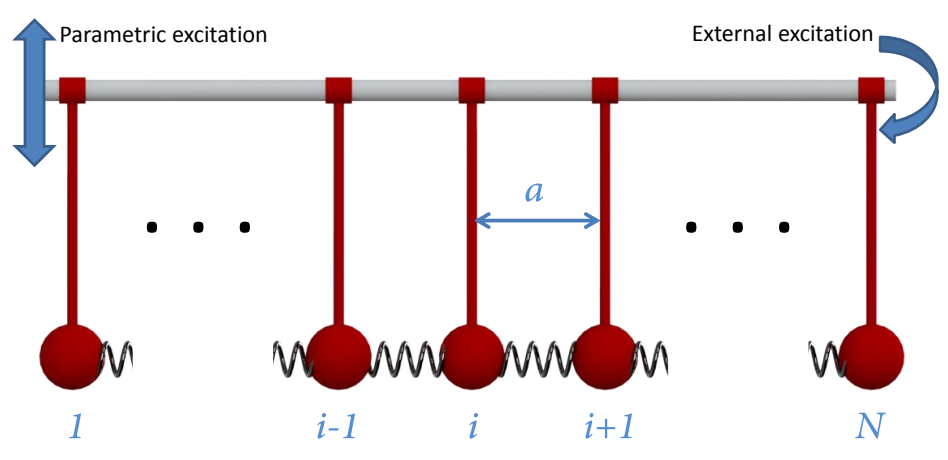

(b)

FIGURE 1: Array of coupled pendulums under simultaneous parametric and external excitations.

where $L=T-V$ is the Lagrangian of the system and $Q_{n}$ is the nonconservative generalized forces applied to the $n^{\text {th }}$ pendulum (the sum of the friction force and the external force). Hence, the $n^{\text {th }}$ pendulum's equation of motion is determined as follows :

$$
\begin{aligned}
& m l_{n}^{2} \frac{d^{2} \theta_{n}}{d t^{2}}+\alpha l_{n} \frac{d \theta_{n}}{d t}+k_{L}\left(2 \theta_{n}-\theta_{n+1}-\theta_{n-1}\right)=-m l_{n}\left[g+4 A_{e} \omega_{e}^{2} \cos \left(2 \omega_{e} t\right)\right] \sin \left(\theta_{n}\right) \\
& +f \cos \left(\omega_{e} t\right) ; \quad n=1,2, . ., N
\end{aligned}
$$

where $\alpha$ is the damping coefficient. When neglecting the mass of the rigid rod, all pendulums have the same moment of inertia $I=m l_{n}^{2}$, where $l_{n}$ is the length of the $n^{\text {th }}$ pendulum.

The considered system is excited by two forces at the drive frequency $\omega$ : an external force $f \cos (\omega t)$ applied to one or several pendulums, and a parametric force $4 A_{e} \omega^{2} \cos (2 \omega t)$ due to the base excitation of the system.

The boundary conditions associated to Equation (7) are $\theta_{0}=0$ and $\theta_{N+1}=0$. By expanding $\sin \left(\theta_{n}\right)$ in Taylor series up to the third order, Equation (7) can be written as :

$$
\begin{aligned}
& m l_{n}{ }^{2} \frac{d^{2} \theta_{n}}{d t^{2}}+\alpha l_{n} \frac{d \theta_{n}}{d t}+k_{L}\left(2 \theta_{n}-\theta_{n+1}-\theta_{n-1}\right)=-m l_{n}\left[g+4 A_{e} \omega_{e}^{2} \cos \left(2 \omega_{e} t\right)\right]\left(\theta_{n}-\frac{1}{6} \theta_{n}^{3}\right) \\
& +f \cos \left(\omega_{e} t\right) ; \quad n=1,2, . ., N
\end{aligned}
$$

Equation (8) governs the dynamics of a chain of Duffing oscillators linearly coupled by the spring coefficient $k_{L}$ and subjected to simultaneous external and parametric excitations. 


\section{Nonlinear energy localization}

\subsection{Nonlinear Schrodinger equation}

In order to investigate the effects of an external excitation on the nonlinear localization phenomena in a chain of coupled pendulums, we use the multiple scales method on Equation (8) to derive the nonlinear Schrodinger equation. To do so, we make the following assumption, where $\omega_{0} \approx \sqrt{\frac{g}{l}}$ and $\varepsilon$ is a small nondenominational fixed parameter :

$$
\begin{array}{llrl}
\omega & =\omega_{0}\left(1-\frac{\varepsilon^{2}}{2}\right) & \omega^{2} \approx \omega_{0}^{2}\left(1-\varepsilon^{2}\right) & 4 A_{e} \omega^{2}=2 h \varepsilon^{2} \\
\alpha=\gamma \varepsilon^{2} & k_{L}=\varepsilon^{2} k & F=2 f \varepsilon^{3}
\end{array}
$$

The displacement of the $n^{n t}$ resonator can be written as :

$$
\theta_{n}(T)=2 \varepsilon\left[\psi_{n}(T) e^{-i \omega t}+\bar{\psi}_{n}(T) e^{i \omega t}\right]
$$

where $T=\frac{\varepsilon^{2}}{2} t$ and $\Psi_{n}$ is an unknown complex function. Therefore

$$
\begin{gathered}
\dot{\theta}_{n}=\varepsilon^{3} \frac{\partial \psi_{n}}{\partial T} e^{-i \omega t}-2 i \omega \varepsilon \psi_{n} e^{-i \omega t}+c c \\
\ddot{\theta}_{n}=-2 i \omega \varepsilon^{3} \frac{\partial \psi_{n}}{\partial T} e^{-i \omega t}-2 \omega^{2} \varepsilon \psi_{n} e^{-i \omega t}+c c \\
\theta_{n}^{3}=8 \varepsilon^{3}\left[\psi_{n}^{3}(T) e^{-3 i \omega t}+3 \psi_{n}^{2} \bar{\psi}_{n}(T) e^{-i \omega t}+c c\right]
\end{gathered}
$$

where $c c$ corresponds to the complex conjugate. Substituting Equations (9-12) into equation (8), we obtain :

$$
\begin{aligned}
& m l^{2}\left(-2 i \omega \varepsilon^{3} \frac{\partial \psi_{n}}{\partial T} e^{-i \omega t}-2 \omega^{2} \varepsilon \psi_{n} e^{-i \omega t}\right) \\
& +\gamma l \varepsilon^{2}\left(\varepsilon^{3} \frac{\partial \psi_{n}}{\partial T} e^{-i \omega t}-2 i \omega \varepsilon \psi_{n} e^{-i \omega t}\right) \\
& +2 \varepsilon^{3} k\left(2 \psi_{n} e^{-i \omega t}-\psi_{n-1} e^{-i \omega t}-\psi_{n+1} e^{-i \omega t}\right)= \\
& -m \lg \left(2 \varepsilon \psi_{n} e^{-i \omega t}-\frac{8}{6} \varepsilon^{3} \psi_{n}^{3} e^{-3 i \omega t}-4 \varepsilon^{3} \psi_{n}^{2} \bar{\psi}_{n} e^{-i \omega t}\right) \\
& -2 h \varepsilon^{3} m l \bar{\psi}_{n} e^{-i \omega t}+f \varepsilon^{3} e^{-i \omega t}
\end{aligned}
$$

Keeping the terms proportional to $e^{-i \omega t}$ and $\varepsilon^{3}$, we obtain :

$$
\begin{aligned}
& -2 i \omega_{0} m l^{2} \frac{\partial \psi_{n}}{\partial T}+2 \omega_{0}^{2} m l^{2} \psi_{n}-2 i \omega_{0} \gamma l \psi_{n} \\
& +2 k\left(2 \psi_{n}-\psi_{n-1}-\psi_{n+1}\right)=4 m l g \psi_{n}^{2} \bar{\psi}_{n}-2 h m l \bar{\psi}_{n}+f
\end{aligned}
$$


Equation (14) can be written as :

$$
\begin{aligned}
& i \frac{\partial \psi_{n}}{\partial T}-\omega_{0} \psi_{n}-\frac{k}{\omega_{0} m l^{2}}\left(2 \psi_{n}-\psi_{n-1}-\psi_{n+1}\right) \\
& +2 \frac{g}{\omega_{0} l} \psi_{n}^{2} \bar{\psi}_{n}=\frac{h}{\omega_{0} l} \bar{\psi}_{n}-i \frac{\gamma}{m l} \psi_{n}-\frac{f}{2 \omega_{0} m l^{2}}
\end{aligned}
$$

By choosing $\tau=\omega_{0} T$, Equation (15) becomes :

$$
\begin{aligned}
& i \frac{\partial \psi_{n}}{\partial \tau}-\psi_{n}-\frac{k}{\omega_{0}^{2} m l^{2}}\left(2 \psi_{n}-\psi_{n-1}-\psi_{n+1}\right) \\
& +2 \psi_{n}^{2} \bar{\psi}_{n}=\frac{h}{\omega_{0}^{2} l} \bar{\psi}_{n}-i \frac{\gamma}{m l \omega_{0}} \psi_{n}-\frac{f}{2 \omega_{0}^{2} m l^{2}}
\end{aligned}
$$

Equation (16) is called the discrete nonlinear Schrodinger equation. In order to write it in its continuous form, we introduce the spatial variable $x$ so that $\psi_{n}=\psi\left(x_{n}\right)$ where $x_{n+1}=x_{n}+\Delta x$ The Taylor expansion of $\psi_{n}$ gives :

$$
\psi_{n \pm 1}=\psi_{n} \pm \psi^{\prime} \Delta x+\frac{(\Delta x)^{2}}{2} \psi^{\prime \prime}
$$

so

$$
\psi_{n+1}+\psi_{n-1}-2 \psi_{n}=(\Delta x)^{2} \psi^{\prime \prime}
$$

where $\psi^{\prime}=\frac{\partial \psi}{\partial x}$ and $\psi^{\prime \prime}=\frac{\partial^{2} \psi}{\partial x^{2}}$. We set $(\Delta x)^{2}=\frac{\omega_{0}^{2} m l^{2}}{k} ; H=\frac{h}{\omega_{0}^{2} l} ; \Gamma=\frac{\gamma}{m l \omega_{0}}$ and $F_{e}=\frac{f}{2 \omega_{0}^{2} m l^{2}}$.

Finaly, Equation (16) can be written as :

$$
i \psi_{\tau}+\psi_{x x}-\psi+2|\psi|^{2} \psi=H \bar{\psi}-i \Gamma \psi-F_{e}
$$

Equation (19) is the continuous nonlinear damped Schrodinger equation (NLS) with external and parametric excitations. When $F_{e}=0$, we obtain a parametrically driven damped nonlinear Schrdinger equation, which can be used to study intrinsic localized modes in arrays of coupled pendulums [14, 22] or arrays of coupled microelectromechanical systems [7] and to model parametrically driven media in hydrodynamics [23] and optics [24, 25].

\subsection{Intrinsic localized modes}

We start by solving the nonlinear Schrodinger equation without damping $(\Gamma=0)$. Equation (19) exhibits two stationary soliton solutions [26] :

$$
\psi(x, t)=\psi_{ \pm}(x)=\psi_{0}\left(1+\frac{2 \sinh ^{2} \eta}{1 \pm \cosh (\eta) \cosh (A x)}\right)
$$

where $\eta$ is defined by 


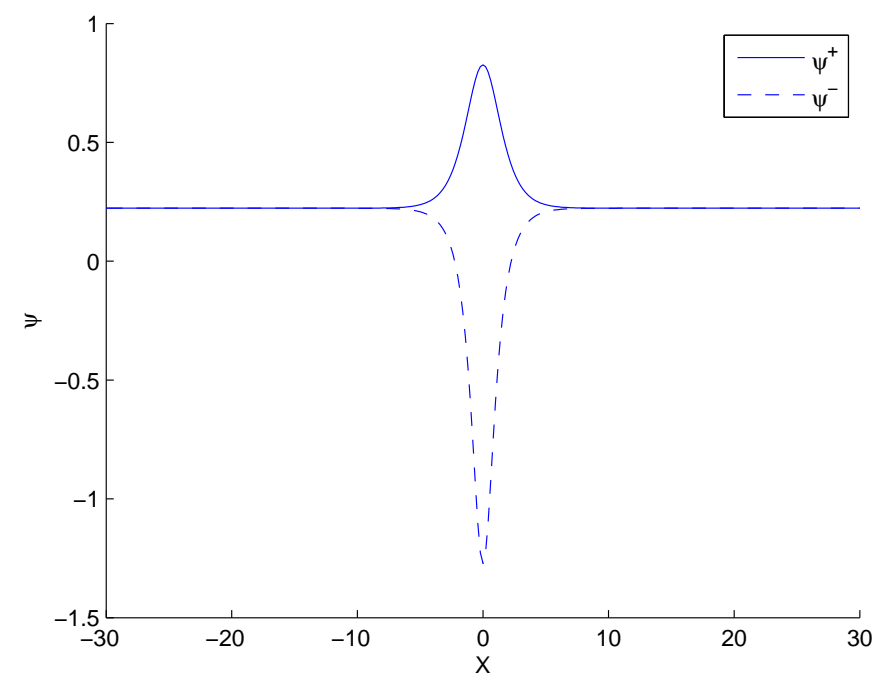

Figure 2: Soliton $\psi_{ \pm}(x)$ for the undamped case $\Gamma=0 . \eta=1.5 ; F_{e}=0.2454 ; H=0.2$

$$
\mathscr{H}(\eta)=\frac{\sqrt{2} \cosh ^{2} \eta}{\left(1+2 \cosh ^{2} \eta\right)^{3 / 2}}=\frac{F_{e}}{(1+H)^{3 / 2}}
$$

$\mathscr{H}(\eta)$ being a monotonically decreasing function, $\eta$ is uniquely determined by $\mathscr{H} . A / 2$ is the area of $\psi_{+}$and $\psi_{-}$solitons :

$$
A=2 \int\left(\psi_{ \pm}^{2}(x)-\psi_{0}^{2}\right) d x=2 \sinh (\eta) \psi_{0}
$$

and $\psi_{0}$ is the asymptotic value of $\psi_{ \pm}$

$$
\psi_{ \pm}(x) \underset{x \rightarrow \infty}{\rightarrow} \psi_{0} \quad \psi_{0}^{3}=\frac{F}{4 \cosh ^{2} \eta}
$$

Figure 2 presents the two exact soliton solutions $\psi_{ \pm}(x)$ with the expression (20). The two solutions exist when $\eta>0$ that corresponds to $\frac{F_{e}}{(1+H)^{3 / 2}}<\sqrt{\frac{2}{27}}$

For $\Gamma>0$, no exact solutions are available. Therefore, we use the continuous analog Newton's method to obtain numerically a soliton solution for Equation (19). The discretized Schrodinger equation is written as :

$$
G(\Psi)=0
$$


Where $\Psi=\left(\psi_{0}, \psi_{1}, \ldots, \psi_{N+1}\right)$ is the discretized solution, with $\psi_{n}=\psi\left(x_{n}\right)$ and $x_{n}=-\frac{L}{2}+n \Delta x$. Therefore, $G_{n}$ could be written as :

$$
\begin{aligned}
& G_{n}=\frac{\psi_{n+1}+\psi_{n-1}-2 \psi_{n}}{(\Delta x)^{2}}-\psi_{n}+2\left|\psi_{n}\right|^{2} \psi_{n}+i \Gamma \psi_{n} \\
& -H \bar{\psi}_{n}+F_{e} \psi_{n} \quad ; n=1,2, \ldots, N
\end{aligned}
$$

To define $G_{0}$ and $\left.G_{(} N+1\right)$, we use the open-end boundary condition $\psi_{x}\left( \pm \frac{L}{2}\right)=0$ coupled with the second order finite difference approximation of $\psi_{x}$ therefore :

$$
G_{0}=\frac{-3 \psi_{0}+4 \psi_{1}-\psi_{2}}{2 \Delta x} \quad G_{N+1}=\frac{\psi_{N-1}-4 \psi_{N}+3 \psi_{N+1}}{2 \Delta x}
$$

Newtons method is one of the commonest methods to solve Equation (21) by introducing the auxiliary parameter $\xi$ in such a way that $\Psi$ satisfies the differential equation :

$$
\frac{\partial}{\partial \xi} G(\Psi(\xi))+G(\Psi(\xi))=0
$$

With the initial condition $\Psi(0)=\Psi^{(0)}$, where $\Psi^{(0)}$ is an initial guess for the soliton solution. Equation (23) could be transformed to :

$$
\Psi^{(i+1)}=\Psi^{(i)}-\left(\frac{\partial G}{\partial x}\right)_{\Psi^{(i)}}^{-1} G\left(\Psi^{(i)}\right)
$$

Where $i=0,1, \ldots$ until we minimize the error $\delta$, which is defined as :

$$
\delta^{(i)}=\max _{1 \leq n \leq N}\left\{\left|\operatorname{Re}\left(G_{n}\left(\Psi^{(i)}\right)\right)\right|,\left|\operatorname{Im}\left(G_{n}\left(\Psi^{(i)}\right)\right)\right|\right\}
$$

To obtain a numerical solution of Equation (19), the following steps are used :

1. Choose $\Psi^{(0)}$, the exact solution of NLS without damping $(\Gamma=0)$, to be the initial approximation for the NLS with damping $(\Gamma=0.01)$.

2. Calculate $\Psi^{(i+1)}$ and $\delta^{(i)}$ using Equations (24) and (25) until we minimize the error $\delta^{(i)}$.

3. After computing the solution for the case $\Gamma=0.01$, choose this solution as the initial approximation for the equation with the incremented value of $\Gamma$.

4. Compute the numerical solution of Equation (19) as we did in step 2.

5. Keep doing steps 3 and 4 until reaching the desired value of $\Gamma$. 

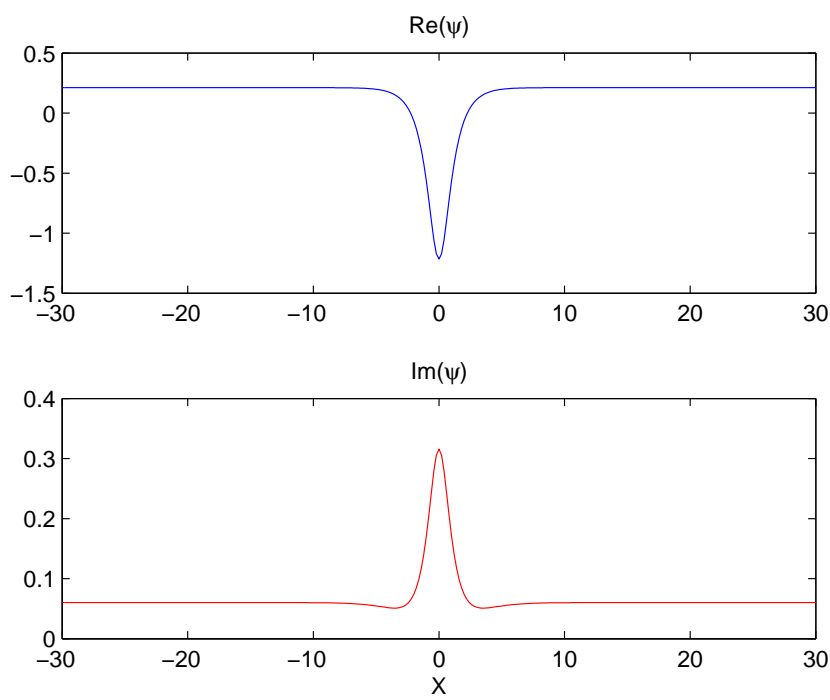

FIGURE 3: Soliton solution in the damped case $\Gamma=0.2 \eta=1.5 ; F_{e}=0.2454 ; H=0.2 ; \Gamma=0.2$

The final vector $\Psi$ of this algorithm is the soliton solution of the NLS equation with the desired damping $\Gamma$. Figure 3 displays the real and the imaginary parts of the soliton solution after applying the previous algorithm. Unlike the undamped NLS equation and its exact solution given by Equation (20), the imaginary part of the soliton solution becomes nonzero when we add a non-null damping coefficient $\Gamma$.

\section{Numerical simulations and discussion}

After showing that the equation of motion (8) can be transformed to a nonlinear Schrodinger equation, numerical simulations are performed on the discretized equation (16) from $x=-L / 2$ to $x=L / 2$. We choose $L=60$ and the spacing between the oscillators $\Delta x=0.2$ which leads to a total number of oscillators $N_{p e n}=301$. The system of nonlinear differential equations is numerically solved using Runge-Kutta method and $\Psi$ is used as an initial condition for Schrodinger equation.

When no external force is applied to the array of pendulums, Equation (16) becomes a parametrically excited undamped nonlinear Schrodinger equation. Barashenkov et al. [27] showed that this equation has two stationary solutions $\psi_{+}$and $\psi_{-}$where $\psi_{-}$is always unstable. The chart of stability of $\psi_{+}$is depicted in Figure 4 and it includes three main regions : 


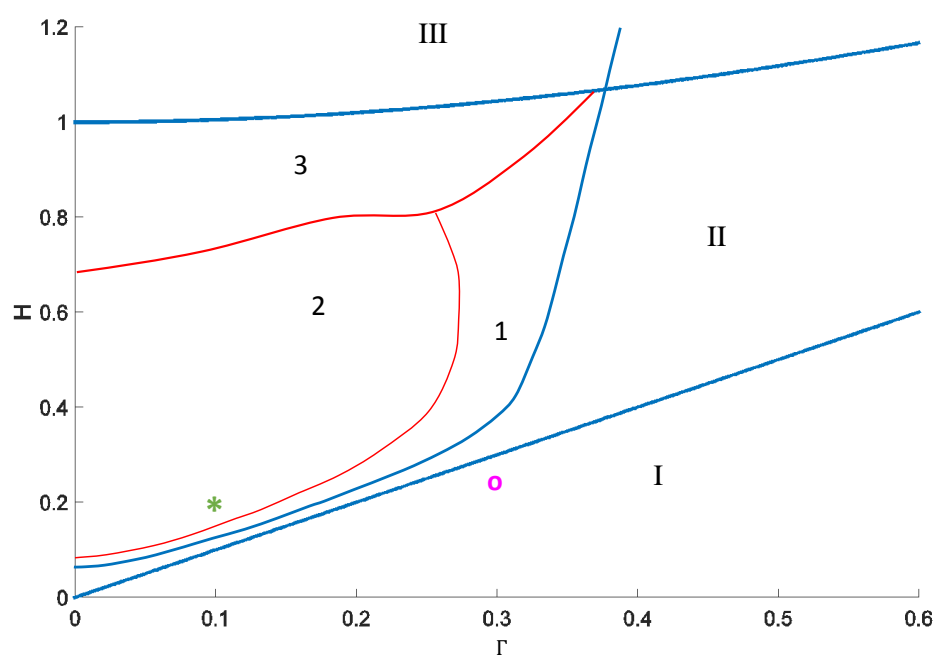

Figure 4: Attractor chart of the continuous NLS equation $(19)$ on the $(\Gamma ; H)$ plane $\left(F_{e}=0\right)$ [27].

— In region "I" localization phenomena is impossible and the only existing solution is zero attractor.

— In region "II" the soliton $\psi_{+}$is a stable solution.

— In region "III" the soliton $\psi_{+}$exist but it is unstable.

- The remaining regions are unstable with respect to a local mode :

* region "1" denotes temporally periodic solutions.

* region "2" marks the area where the only attractor is $\psi=0$.

* region "3" presents the domain of spatio-temporal chaos.

Figures $5(\mathrm{a}, \mathrm{b})$ display the time series and phase portrait for the central oscillator $\psi_{0}$ while Figure 5(c) shows the evolution of the soliton solution with respect to time. It is clearly seen that oscillations cease as the real and imaginary parts of $\psi_{0}$ converge to a fixed point $\psi_{0}=0$, which presents an asymptotic stable spiral point. These plots correspond to the point with coordinates $(\Gamma=0.1 ; H=0.2)$ marked by a star in Figure 4. This point is in region "2" which corresponds to the trivial solution $\psi(x, t) \equiv 0$.

Next, a Schrodinger equation under external excitation can be obtained by cancelling the parametric excitation. This equation is numerically solved and the initial condition is determined by applying the continuous analog Newton's method for $\Gamma=0.1 ; H=0 ; F=0.166$. It is shown in 


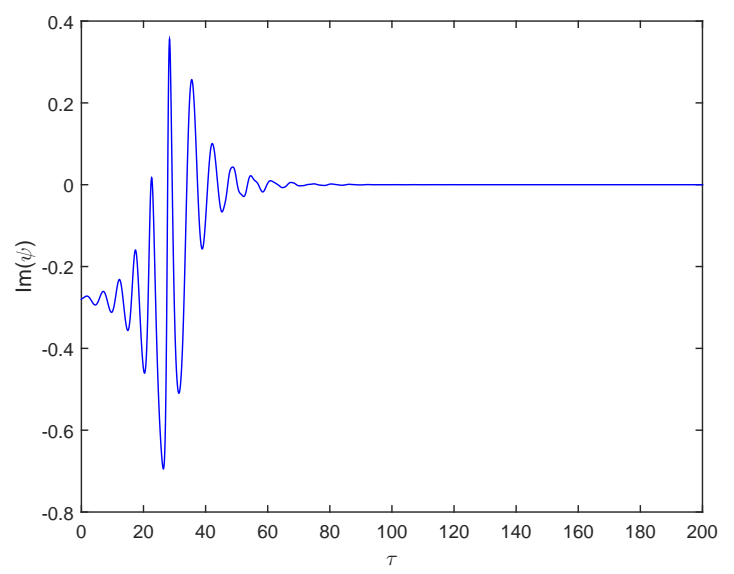

(a)

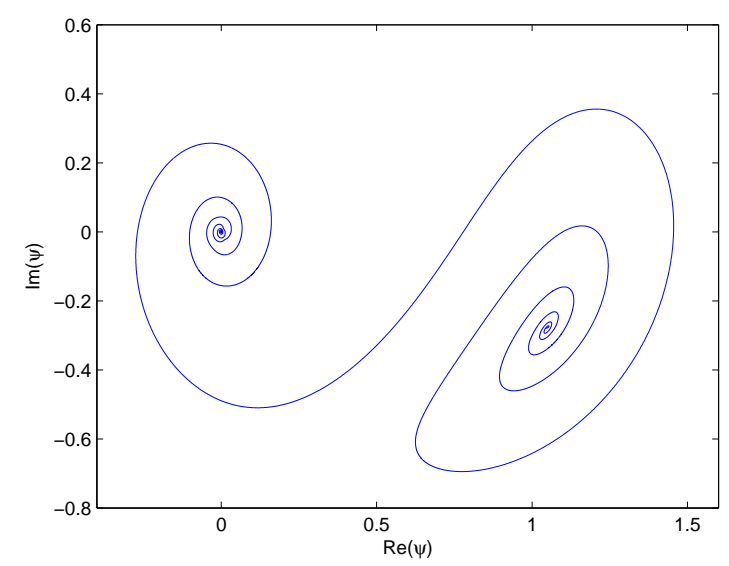

(b)

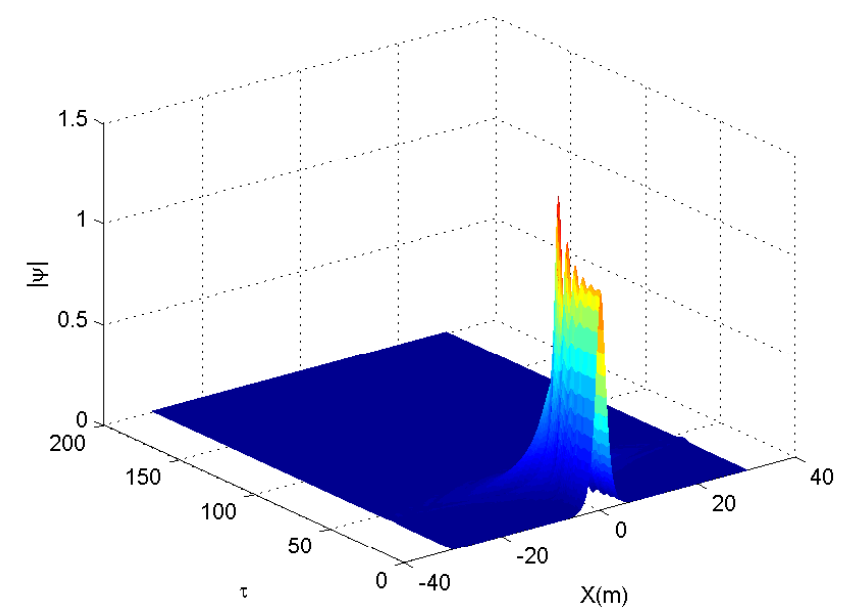

(c)

FIGURE 5: Time series and phase portrait of the soliton solution of the Schrodinger equation (19) with pure parametric excitation $\left(F_{e}=0\right)$. Only the trivial solution $\Psi=0$ found. Here $\Gamma=0.1 ; H=0.2 ; F_{e}=0$ 
Figure 6 that the same behavior as the previous case is obtained. Nevertheless, the soliton solution converges to a constant solution $\Psi \equiv$ cst due to the effect of the external force.

Truly, when the array of pendulums is excited with either a parametric or external force, we did not have a soliton solution, while a remarkable formation of a stable soliton is notable when the two type of forces are combined which is illustrated in Figure 7. As shown by the phase portrait for the central oscillator $\psi_{0}$, the oscillations of the amplitude are regular, and we observe a period-2 limit cycle.

Interestingly, if an external force is added to the system $\left(\Gamma=0.3 ; H=0.25 ; F_{e}=0.23\right)$, the amplitude of the central oscillator $\psi_{0}$, presented in Figure 8, converges to a constant value when $t$ goes to infinity. The point in the phase portrait, where the real and imaginary parts converge to, corresponds to an asymptotic stable spiral point. It is clearly seen that the amplitude of the soliton solution maintains the same shape with respect to time which confirms the stability of the obtained intrinsic localized mode. Thus, the external force can be used as a tuning parameter to modify the existence and stability of solitons in parametrically driven coupled pendulums. This remarkable result can be exploited in order to control the nonlinear localization phenomenon in periodic structures.

\section{Conclusions}

In this paper, we investigated the energy localization phenomenon in an array of coupled pendulums under simultaneous external and parametric excitations by means of a nonlinear Schrodinger equation. The latter is obtained using the multiple scale technique which has been applied on the system of coupled nonlinear equations of motion. The stationary solution of the undamped nonlinear Schrodinger equation was determined analytically and based on this solution, the stationary solution for the damped case was numerically computed using the continuous analog Newton's method. Several numerical simulations have been performed on the Schrodinger equation using the Runge-Kutta method and the stationary solution as an initial condition. We demonstrated that the use of external and parametric excitations simultaneously enables the transformation of a zero attractor soliton solution to a periodically stable one. Therefore, by adding an external 


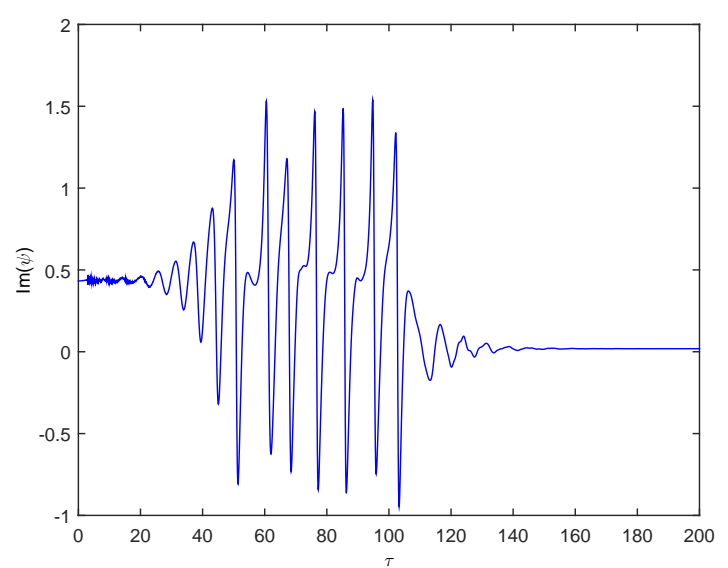

(a)

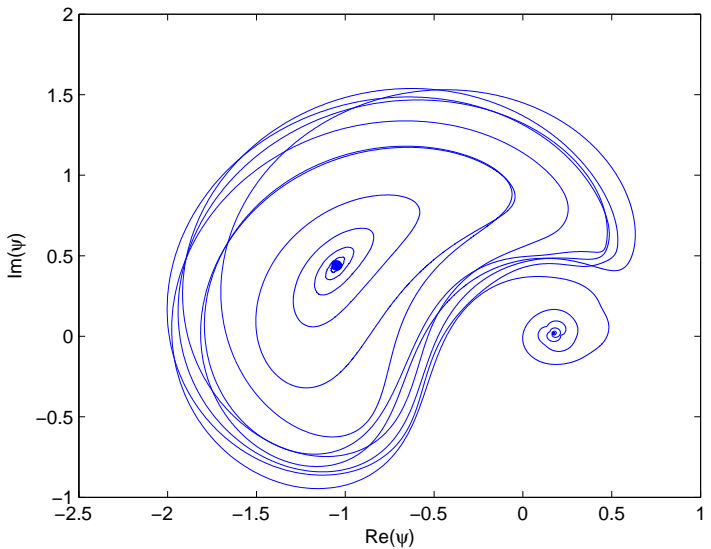

(b)

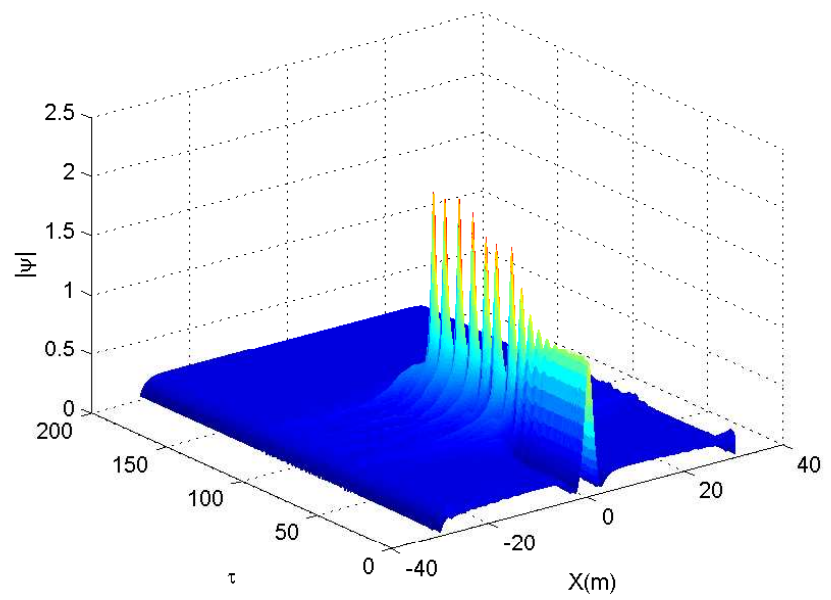

(c)

FIGURE 6: Time series and phase portrait of the soliton solution of the Schrodinger equation (19) with pure external excitation $\Gamma=0.1 ; H=0 ; F_{e}=0.166$ 


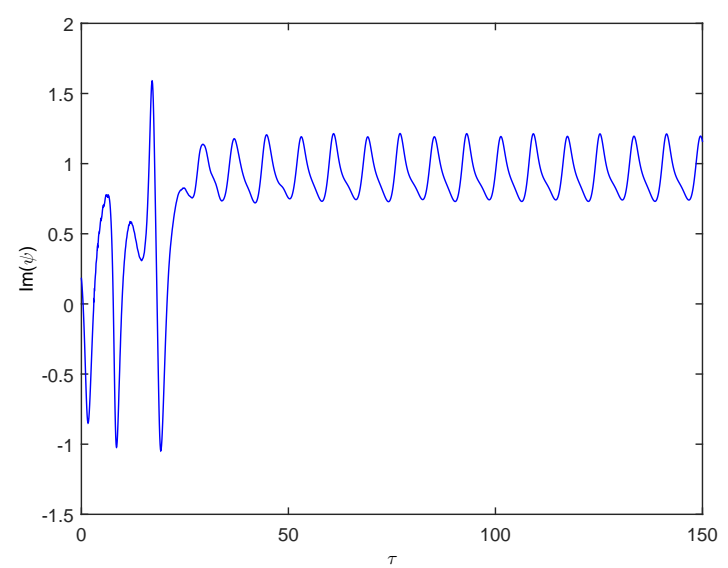

(a)

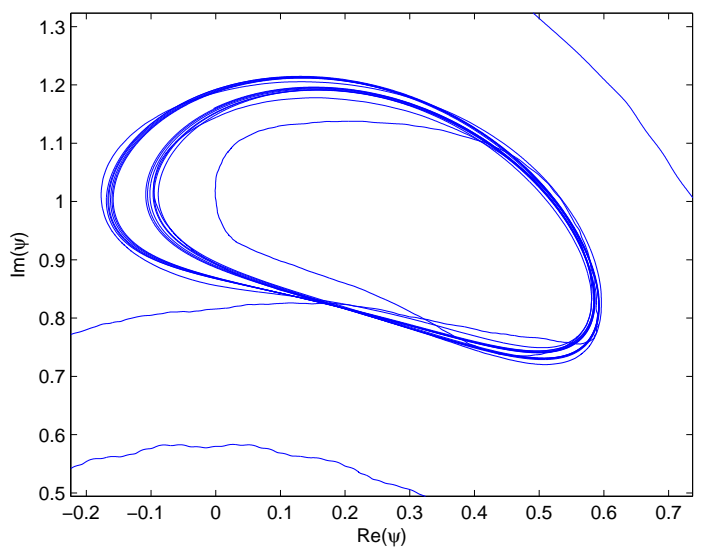

(b)

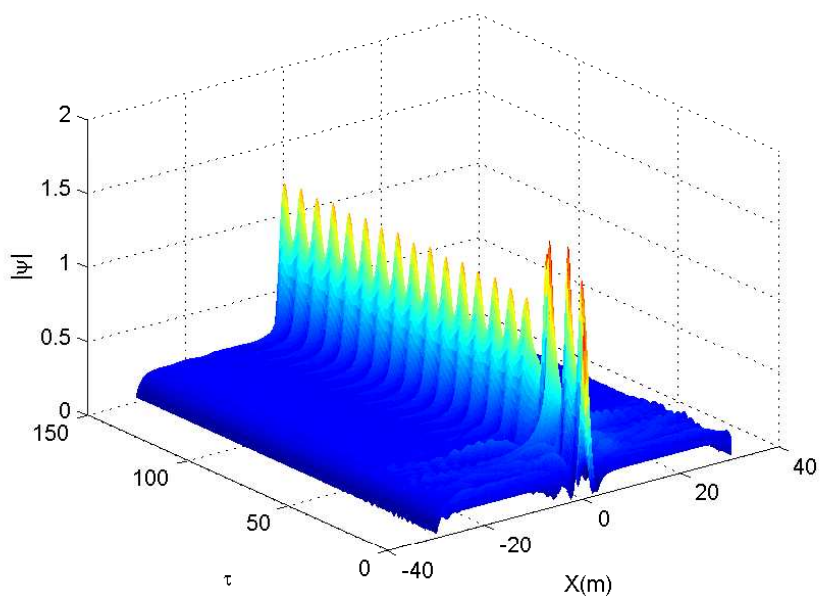

(c)

Figure 7: Time series and phase portrait of the soliton solution of the Schrodinger equation (19) with two excitations, external and parametric. Here $\Gamma=0.1 ; H=0.2 ; F_{e}=0.166$ 


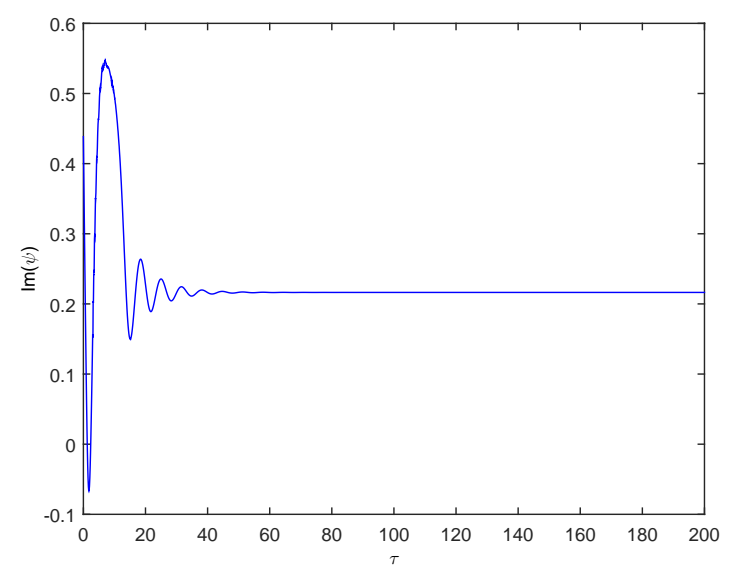

(a)

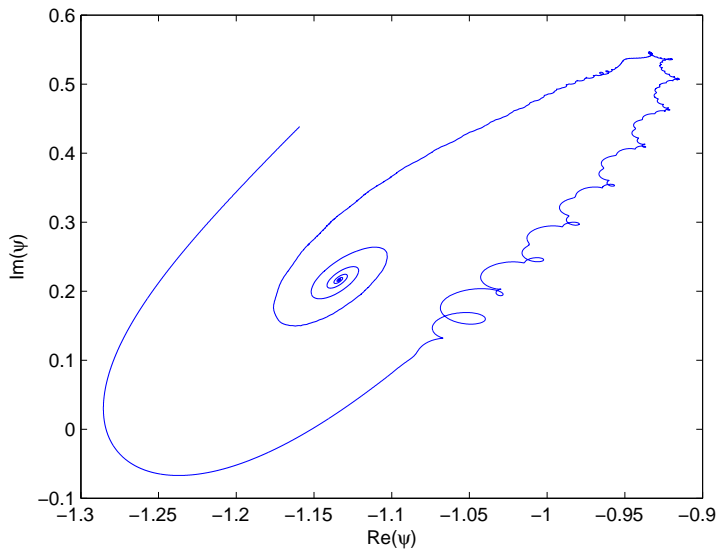

(b)

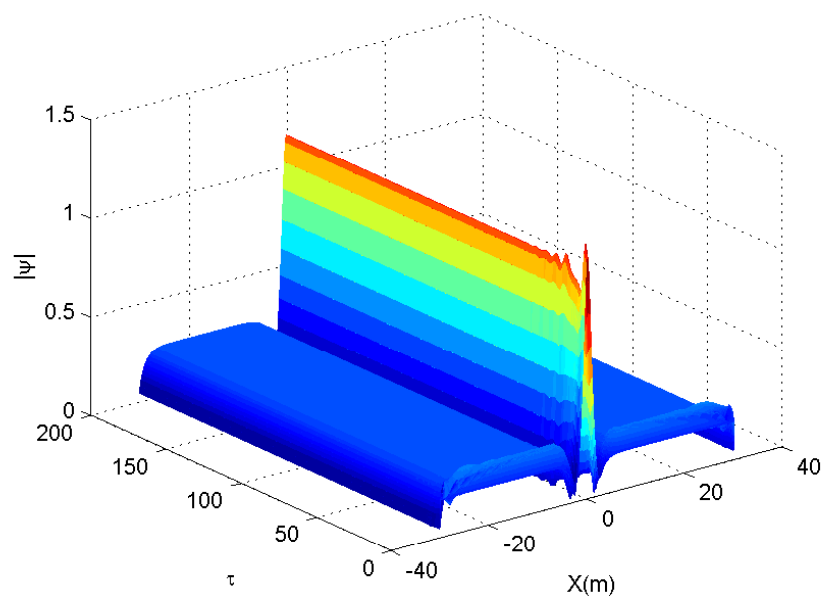

(c)

Figure 8: Time series and phase portrait of the soliton solution of the Schrodinger equation (19) with two excitation, external and parametric. Here $\Gamma=0.3 ; H=0.25 ; F_{e}=0.231$ 
excitation one can increase the existence region of solitons. In practice, this stabilization mechanism opens the way towards the control of the solitons in damped nonlinear periodic lattices for efficient energy transport applications.

\section{Acknowledgments}

This project has been performed in cooperation with the Labex ACTION program (contract ANR-11-LABX-01-01).

\section{Références}

[1] Wu J, Keolian R, Rudnick I. Observation of a nonpropagating hydrodynamic soliton. Physical review letters. 1984 ;52(16) :1421-1424.

[2] Denardo B, Wright W, Putterman S, Larraza A. Observation of a kink soliton on the surface of a liquid. Phys Rev Lett. 1990 Mar;64:1518-1521.

[3] Wang X, Wei R. Dynamics of Multisoliton Interactions in Parametrically Resonant Systems. Phys Rev Lett. 1997 Apr ;8 :2744-2747.

[4] Vasanthi CC, Latha MM. Heisenberg ferromagnetic spin chain with bilinear and biquadratic interactions in (2 + 1) dimensions. Communications in Nonlinear Science and Numerical Simulation. 2015 ;28(13):109 - 122.

[5] Barashenkov I, Bogdan M, Korobov V. Stability diagram of the phase-locked solitons in the parametrically driven, damped nonlinear Schrödinger equation. EPL (Europhysics Letters). $1991 ; 15(2): 113$.

[6] Floría LM, Mazo JJ. Dissipative dynamics of the Frenkel-Kontorova model. Advances in Physics. $1996 ; 45(6): 505-598$.

[7] Kenig E, Malomed BA, Cross MC, Lifshitz R. Intrinsic localized modes in parametrically driven arrays of nonlinear resonators. Phys Rev E. 2009 Oct;80:046202.

[8] Ikeda T, Harata Y, Nishimura K. Intrinsic Localized Modes of Harmonic Oscillations in Pendulum Arrays Subjected to Horizontal Excitation. Journal of Computational and Nonlinear Dynamics. 2015 ;10(2):021007.

[9] Ikeda T, Harata Y, Nishimura K. Intrinsic Localized Modes of Harmonic Oscillations in Nonlinear Oscillator Arrays. Journal of Computational and Nonlinear Dynamics. 2013 ;8(4) :041009.

[10] Ivancevic VG, Ivancevic TT. Sine-Gordon Solitons, Kinks and Breathers as Physical Models of Nonlinear Excitations in Living Cellular Structures. Journal of Geometry and Symmetry in Physics. 2013 ;31 :1-56.

[11] Khomeriki R, Leon J. Tristability in the pendula chain. Physical Review E. 2008 ;78(5):057202.

[12] Braiman Y, Lindner JF, Ditto WL. Taming spatiotemporal chaos with disorder. Nature. 1995 ;378(6556) :465467. 
[13] Qi F, Hou Z, Xin H. Ordering chaos by random shortcuts. Physical review letters. 2003 ;91(6):064102.

[14] Alexeeva N, Barashenkov I, Tsironis G. Impurity-induced stabilization of solitons in arrays of parametrically driven nonlinear oscillators. Physical review letters. 2000 ;84(14):3053.

[15] Chen W, Zhu Y, Lu L. Observations of impurity-soliton interactions in driven Frenkel-Kontorova chains. Physical Review B. $2003 ; 67(18): 184301$.

[16] Hai-Qing X, Yi T. Parametrically Driven Solitons in a Chain of Nonlinear Coupled Pendula with an Impurity. Chinese Physics Letters. 2006 ;23(6):1544.

[17] Chen W, Hu B, Zhang H. Interactions between impurities and nonlinear waves in a driven nonlinear pendulum chain. Physical Review B. $2002 ; 65(13): 134302$.

[18] Zhu Y, Chen W, Lü L. Experiments on the interactions between impurities and solitary waves in lattice model. Science in China Series G : Physics, Mechanics and Astronomy. $2003 ; 46(5): 460-467$.

[19] Collective dynamics of periodic nonlinear oscillators under simultaneous parametric and external excitations. Nonlinear Dynamics. $2015 ; 82(1-2)$.

[20] Kacem N, Baguet S, Dufour R, Hentz S. Stability control of nonlinear micromechanical resonators under simultaneous primary and superharmonic resonances. Applied Physics Letters. 2011 ;98(19).

[21] Kacem N, Baguet S, Duraffourg L, Jourdan G, Dufour R, Hentz S. Overcoming limitations of nanomechanical resonators with simultaneous resonances. Applied Physics Letters. $2015 ; 107(7)$.

[22] Thakur RB, English LQ, Sievers AJ. Driven intrinsic localized modes in a coupled pendulum array. Journal of Physics D : Applied Physics. 2008 ;41(1):015503.

[23] Zhang W, Viñals J. Secondary Instabilities and Spatiotemporal Chaos in Parametric Surface Waves. Phys Rev Lett. 1995 Jan ; 74:690-693.

[24] Longhi S. Stable multipulse states in a nonlinear dispersive cavity with parametric gain. Phys Rev E. 1996 May;53:5520-5522.

[25] Radha R, Vinayagam PS, Sudharsan JB, Malomed BA. Persistent bright solitons in sign-indefinite coupled nonlinear Schrdinger equations with a time-dependent harmonic trap. Communications in Nonlinear Science and Numerical Simulation. $2016 ; 31(13): 30-39$.

[26] Barashenkov I, Smirnov YS. Existence and stability chart for the ac-driven, damped nonlinear Schrödinger solitons. Physical Review E. 1996 ;54(5):5707.

[27] Bondila M, Barashenkov IV, Bogdan MM. Topography of attractors of the parametrically driven nonlinear Schrödinger equation. Physica D : Nonlinear Phenomena. 1995 ;87(1) :314-320. 